Die als «Rede und Gegenrede» konzipierte Diskussion zwischen dem Philosophen Otfried Höffe und dem Theologen Johannes Fischer über zentrale Fragen der Ethik - auch für Mediziner und die Medizin - wird mit den folgenden Beiträgen der beiden Autoren abgeschlossen. Bereits erschienen sind «Philosophische Ethik: Fahne im Wind oder Fels in der Brandung?» [1], «Ethik als Prinzipienethik?» [2] und «Narrative Ethik als Alternative?» [3].

Die Redaktion

1 Höffe O. Philosophische Ethik: Fahne im Wind oder Fels in der Brandung? Schweiz Ärztezeitung. 2010;91(32):1199-1202.

2 Fischer J. Ethik als Prinzipienethik? Schweiz Ärztezeitung. 2010;91(38):1484-7.

3 Höffe O. Narrative Ethik als Alternative? Schweiz Ärztezeitung 2010;91(38):1488-9.

\title{
Der narrative Gehalt moralischer Begriffe
}

\section{Johannes Fischer}

Professor für Theologische Ethik, Leiter des Instituts für Sozialethik und verantwortlicher Leiter des universitären Forschungsschwerpunktes Ethik am Ethik-Zentrum der Universität Zürich
Korrespondenz:

Prof. Dr. theol. Johannes Fischer

Universität Zürich

Institut für Sozialethik

Zollikerstrasse 117

CH-8008 Zürich

fischer@sozethik.uzh.ch
Es gehe darum, «die Vielfalt der Phänomene auf wenige Begriffe, im Fall einer klaren Gemeinsamkeit sogar auf einen einzigen Begriff zu bringen». Hierin sieht Otfried Höffe die spezifische Leistung der Moralphilosophie und zugleich die Alternative zu dem, was er «narrative Ethik» nennt - ein Ausdruck, den ich bewusst vermeide, da er irreführend ist. Ohne Zweifel: Ethik hat es wesentlich mit der Bildung und Klärung von Begriffen zu tun hat. Die Frage ist jedoch, ob sie dabei nicht unweigerlich mit der narrativen Verfasstheit der Moral konfrontiert ist. Gibt es doch keinen einzigen moralischen Begriff, der sich rein begrifflich explizieren lässt, d.h. ohne Rekurs auf die Anschauung oder Vorstellung von Situationen oder Handlungen, wie sie sprachlich durch Narrative artikuliert wird.

Nehmen wir den Begriff der Menschenwürde. Ersichtlich lässt sich der Gehalt dieses Begriffs nicht ohne den Rekurs auf die Anschauung bzw. Vorstellung von Verletzungen bestimmen, die Menschen zugefügt werden können. Dieser Rekurs ist zum Beispiel wichtig im Blick auf Versuche, den Gehalt dieses Begriffs auf einen einzigen Aspekt zu reduzieren. So ist von verschiedenen Philosophen der Vorschlag gemacht worden, die Menschenwürde als das Recht aufzufassen, nicht erniedrigt, d.h. in seiner Selbstachtung verletzt zu werden. Ersichtlich ist diese Definition viel zu eng. Wenn im Bosnien-Krieg die serbische Soldateska mit Lastwagen über gefangene Muslime hinweggefahren ist, dann betrachten wir dies zweifellos als eine Menschenwürdeverletzung. Doch soll diese tatsächlich darin bestanden haben, dass die Muslime durch diese Art der Tötung in ihrer Selbstachtung verletzt worden sind? Das mutet wie eine Verharmlosung des Geschehenen an. Die Muslime wurden schliesslich auf brutale Weise umgebracht. Dasselbe lässt sich gegen die bei Kant zu findende Reduktion der Menschenwürde auf den Gedanken der Autonomie geltend machen. Soll sich die Menschenwürdeverletzung in diesem Beispiel tatsächlich darauf beschränken, dass die Autonomie der Muslime missachtet worden ist? Solche Beispiele verdeutlichen, wie problematisch ein begriffliches Denken auf dem Gebiet der Moral ist, das sich von der Anschauung und Vorstellung entfernt. Moralische Begriffe wie Grausamkeit, Erniedrigung oder Menschenwürde sind die sprachliche Artikulation erlebter bzw. narrativ vergegenwärtigter Wirklichkeit, und sie können daher in ihrem Gehalt nur durch den Rückbezug auf diese Wirklichkeit expliziert werden.

Eine rein begriffliche Explikation eines Begriffs wie «Erniedrigung» müsste in der Spezifizierung der relevanten Eigenschaften einer erniedrigenden Handlung bestehen. Zum Beispiel: Erniedrigung liegt vor, wenn jemand einen anderen in seiner Selbstachtung verletzt. Das ist eine deskriptive Feststellung. Lässt sie uns verstehen, was Erniedrigung in moralischer Hinsicht bedeutet, d.h. inwiefern sie ein moralisches Übel ist? Verstehen wir dies nicht erst, wenn wir uns in der Vorstellung vergegenwärtigen, was es für einen Menschen bedeutet, erniedrigt bzw. in seiner Selbstachtung verletzt zu werden? So mag einem jene Szene im Wien der nationalsozialistischen Zeit in den Sinn kommen, als Juden gezwungen wurden, mit Zahnbürsten das Strassenpflaster zu schrubben. Nur über die Vorstellung solcher Szenarien, die uns mit unserer Fähigkeit zur Empathie beanspruchen und die ihre sprachliche Artikulation in Narrativen haben, kommt die moralische Dimension dessen in den Blick, was Erniedrigung heisst. Sie führen mit unbedingter, kategorischer Strenge im Unterschied zu einer blossen Klugheitserwägung vor Augen, dass Menschen nicht erniedrigt werden dürfen. Hier sind wir emotional engagiert. Ich kenne demgegenüber nicht wenige moralphilosophische Untersuchungen, die dem Glauben anhängen, man könne den moralischen Bedeu- 
tungsgehalt von moralischen Begriffen wie «Menschenwürde» desengagiert durch rein begriffliche Explikationen bestimmen (und da geht es nicht um Metaethik). Ich halte dies für eine Verirrung des Denkens, die zur Folge hat, dass die Moralphilosophie sich in begriffliche Abstraktionen verliert und blind wird für die Lebensphänomene [1]. gegenwärtigen, indem sie sie in ihrer Erlebnisqualität vor das innere Auge stellen. Die Narrativität ist also genau diejenige sprachliche Ausdrucksform, die der emotionalen Fundiertheit der Moral entspricht. Der Kern meiner Kritik richtete sich gegen ein prinzipienethisches Denken, wie es in den modernen ethischen Theorien begegnet, das an die Stelle der narrativ the-

\section{Ein begriffliches Denken, das sich von der Anschauung und Vorstellung entfernt, ist auf dem Gebiet der Moral problematisch}

Otfried Höffe macht geltend, dass derjenige, der für die Bedeutung von Emotionen plädiert, asoziale Emotionen wie den Sadismus nicht vergessen dürfe, denen mit Prinzipien wie der Achtung der Menschenwürde begegnet werden muss. Doch wenn der Begriff der Menschenwürde seinen Gehalt aus der Anschauung bzw. Vorstellung von Verletzungen bezieht, die Menschen zugefügt werden können, dann ist er selbst in Emotionen verankert, die bei dieser Anschauung bzw. Vorstellung beteiligt sind. Statt asozialen Emotionen Prinzipien entgegenzusetzen, geht es deshalb darum, die emotionalen Verhaltenseinstellungen zum Gegenstand der Reflexion zu machen, von denen wir uns in unserem Urteilen und Handeln leiten lassen. Eben dies tut das biblische Liebesgebot, um das es in der Samaritererzählung geht.

Es dürfte deutlich sein, dass die These von der narrativen Fundiertheit der Moral nicht als Steckenpferd eines Theologen abgetan werden kann, der «narrative Elemente liebt», was ja auch der heutigen moralphilosophischen Diskussionslage nicht entspricht [2]. Ich betrachte es als ein Defizit von grossen Teilen der heutigen Moralphilosophie, dass sie die Erkenntnis der empirischen Moralforschung in Psychologie und Neurobiologie nicht rezipieren, wonach moralische Wertungen auf der emotionalen Bewertung von Situationen und Handlungen beruhen. Die Bedeutung der Narrativität liegt darin, dass Narrative im Unterschied $\mathrm{zu}$ reinen Deskriptionen Situationen und Handlungen für die emotionale Erkenntnis ver- matisierten, bereits moralisch imprägnierten Wirklichkeit die deskriptiv thematisierte, wertneutrale Wirklichkeit setzt, mit der bekannten Folge des Hiatus zwischen Tatsachen und Wertungen, und das dementsprechend moralische Wertungen aus Geboten - «Geh hin, und tu desgleichen» - Normen oder Prinzipien ableiten muss, die auf diese Wirklichkeit appliziert werden. Der Moralphilosophie fällt dann die Aufgabe der Spezifizierung und Begründung derartiger Prinzipien zu - im Idealfall gar eines einzigen Prinzips. Geht es nicht stattdessen um genaues Hinschauen auf Lebenssituationen - gerade in der Medizin?

\section{Literatur}

1 Vgl. dazu Fischer J. Ethik als rationale Begründung der Moral? Über eine moralphilosophische Verirrung. www.ethik.uzh.ch/ise/publikationen/ publikationen-1.html, Zugriff am 10.8.2010.

Es handelt sich um einen Text, den ich für eine interne Diskussion mit den Philosophinnen und Philosophen am Ethikzentrum der Universität Zürich geschrieben habe.

2 Vgl. Joisten K (Hrsg.). Narrative Ethik. Das Gute und das Böse erzählen. Sonderband 17 der Deutschen Zeitschrift für Philosophie. Berlin; 2007.

In diesem Band finden sich u. a. Texte zu Paul Ricœur und Alasdair MacIntyre, Philosophen, die wichtige Beiträge zum Thema «Narrativität und Moral» geliefert haben. 